\title{
AVALIAÇÃO DAS CULTIVARES DE BANANEIRA PRATA-ANÃ, THAP MAEO E CAIPIRA EM DIFERENTES SISTEMAS DE PLANTIO NO NORTE DE MINAS GERAIS ${ }^{1}$
}

\author{
VALDEIR DIAS GONÇALVES ${ }^{2}$, SILVIANIETSCHE ${ }^{3}$, MARLON CRISTIAN TOLEDO PEREIRA ${ }^{3}$, \\ SEBASTIÃO DE OLIVEIRA E SILVA ${ }^{4}$, TELMA MIRANDA DOS SANTOS ${ }^{5}$, JANAÍNA RIBEIRO OLIVEIRA $^{5}$, \\ LIDIANE RODRIGUES LONDE FRANCO ${ }^{5}$, CARLOS RUGGIERO ${ }^{6}$
}

RESUMO- A banana é uma das frutas tropicais mais consumidas no mundo, respondendo por, aproximadamente, $10 \%$ do comércio mundial de frutas. O objetivo deste trabalho foi avaliar o crescimento e a produção de três cultivares de bananeira sob três sistemas de plantio, em dois ciclos de produção, no período compreendido entre março de 2004 e outubro de 2006, em Janaúba, Minas Gerais. O delineamento experimental foi o de blocos casualizados e duas análises estatísticas: Primeira: 'Prata-Anã' em seis diferentes sistemas de plantio; Segunda: fatorial 2 x $2+1$, com as variedades Caipira e Thap Maeo, em três diferentes sistemas de plantio, com quatro repetições e seis plantas úteis por parcela. Foram avaliadas as seguintes características: altura de planta, circunferência do pseudocaule, número total de folhas, número de folhas vivas na colheita, número de dias do plantio à colheita, massa do cacho, produtividade, número de pencas e número de frutos por cacho. As características avaliadas foram submetidas à análise de variância com desdobramentos das interações significativas, tendo os efeitos dos tratamentos comparados pelo teste de Tukey, a $5 \%$ de probabilidade. A mistura de cultivares e o uso de bordaduras não influenciaram na maioria das características de crescimento, nas três cultivares estudadas. A cultivar Thap Maeo foi superior à 'Caipira' na maioria das características avaliadas, no primeiro e segundo ciclos. Para os sistemas de plantio, foi superior o plantio com uma bordadura. A análise da variedade Prata-Anã dentro dos seis sistemas de plantio apresentou diferença significativa para as características de número de folhas vivas na colheita, no segundo ciclo, número de pencas/cacho e número de frutos/cacho.

Termos para indexação: Musa spp. interplantio, variedades.

\section{EVALUATION OF PRATA ANÃ, THAP MAEO AND CAIPIRA BANANA CULTIVARS IN DIFFERENT PLANTING SYSTEMS IN THE NORTH OF MINAS GERAIS}

\begin{abstract}
Banana is one of the tropical fruits more consumed in the world, answering for approximately $10 \%$ of the world trade of fruits. The purpose of this work was to evaluate the growth and production of three banana cultivars under three planting systems, in two production cycles, in the period from March 2004 to October 2006, in Janaúba, Minas Gerais. The experimental design was in randomized blocks and two statistical analyses: first: Prata Anã in six different planting systems; second: factorial $2 \times 2+1$, with four repetitions and six useful plants per parcel. The evaluated characteristics were: plant height, pseudostem circumference, total number of leaves, number of green leaves in the crop, number of days from planting to harvest, bunch weight, productivity, number of hands and fingers per bunch. The appraised characteristics were submitted to the variance analysis, being the treatments effects compared by the test of Tukey to $5 \%$ of probability. The cultivar mixture and the usage of borders did not influence most of the growth characteristics on the three studied cultivars. The cultivar Thap Maeo was superior to the Caipira in most of the appraised characteristics in the first and second cycles. The planting system with one border was superior. The analysis of the cultivar Prata Anã presented significant difference for the characteristics of green leaves number in the crop in the second cycle, number of hands/ bunch and number of fingers/bunch inside of the six planting systems.
\end{abstract}

Index terms: Musa spp., mixtures, cultivars.

\footnotetext{
${ }^{1}$ Trabalho 109-07). Recebido em: 23-04-2007. Aceito para publicação em: 14-12-2007.Trabalho de Dissertação de Mestrado, Genética e Melhoramento, UNESP, Jaboticabal-SP.

${ }^{2}$ Eng. Agr. Mestrando em Agronomia (Genética e melhoramento de plantas), UNESP, Jaboticabal-SP. e-mail: valdeirdiasgoncalves@yahoo.com.br ${ }^{3}$ Eng. Agr., Dr., Prof., Dep. Ciências Agrárias, UNIMONTES, Cx. Postal 91, CEP 39440-000 Janaúba-MG. e-mail: silvia.nietsche@unimontes.br; marlon.pereira@unimontes.br (Apoio: Finep, Fapemig, CNPq e CAMPO Biotecnologia).

${ }^{4}$ Pesquisador da Embrapa Mandioca e Fruticultura. Cruz das Almas-BA. ssilva@cnpmf.embrapa.br.

${ }_{5}^{5}$ Estudantes do Curso de Agronomia, UNIMONTES, Cx. Postal 91, CEP 39440-000, Janaúba-MG.

${ }^{6}$ Professor da FCAV/UNESP, Jaboticabal-SP. rbf@fcav.unesp.br.
} 


\section{INTRODUÇÃO}

A cultura da bananeira assume importância econômica e social em todo o mundo, sendo cultivada em mais de 80 países tropicais, principalmente por pequenos agricultores. O Brasil é o terceiro maior produtor mundial de banana, com uma produção aproximada de 6,88 milhões de toneladas, em uma área cultivada de 582 mil hectares (FAO, 2007). O mercado interno consome praticamente toda a produção nacional, com um consumo per capita é de 29,0 kg/ano (Gonçalves et al., 2005). Segundo dados da Secretaria de Comércio Exterior, a exportação brasileira de banana, nos nove primeiros meses de 2006 , foi $20,2 \%$ superior ao período de 2005, e, desses totais, a parcela destinada à União Européia aumentou $50 \%$ no valor e na quantidade, enquanto a parcela enviada ao Mercosul caiu 22,7\% (SECEX, 2006).

A bananicultura atingiu, em Minas Gerais, no ano de 2004, uma produção de 36.073 mil cachos, com rendimento médio de 1.115 cachos/ha. A bananicultura irrigada, no norte de Minas Gerais, especialmente nos municípios de Jaíba e Janaúba, apresenta rendimento cerca de $70 \%$ superior à média do Estado, ou seja, 1.900 cachos/ha (Agrianual, 2005). No aspecto social, os projetos de irrigação geram cerca de 12.200 empregos diretos e 24.400 indiretos (Borges \& Oliveira, 2000).

A 'Prata-Anã' é a principal cultivar plantada no norte de Minas Gerais, representando mais de $90 \%$ dos 10.000 ha plantados na região. Desta forma, a economia da região está baseada numa cultivar suscetível a vários problemas fitossanitários, com destaque à Sigatoka- amarela, o que a torna vulnerável por não apresentar resistência a esse fitopatógeno. Recentemente, com a identificação dos primeiros focos da Sigatoka-negra no Estado de Minas Gerais (Castro et al., 2005), a cadeia produtiva da banana tem sentido os impactos da proximidade do patógeno na Região Norte Mineira. Uma das estratégias para a solução dos principais problemas fitossanitários é o desenvolvimento de cultivares resistentes, bem como sua avaliação e caracterização em áreas de produção quando são comparadas às cultivares tradicionais (Silva et al., 2002). O programa de melhoramento genético da bananeira da Embrapa/CNPMF, em Cruz das Almas - BA, está fortemente baseado no desenvolvimento de cultivares do subgrupo Prata, que vêm sendo avaliados nas mais diversas regiões do Brasil. Dentre as cultivares, podemos destacar os híbridos: Pioneira, FHIA 18, SH3640, Pacovan Ken, Tropical, FHIA 01, Garantida, Caprichosa e as cultivares Caipira, Thap Maeo e Prata Baby (Silva et al. 2000, Silva \& Santos-Serejo, 2003). Além do uso das cultivares resistentes, a prática da mistura de cultivares resistentes e suscetíveis em diferentes sistemas de plantio pode servir de barreira física com relação à dispersão dos esporos do fungo, reduzindo a quantidade dos esporos na área, já que esses não irão multiplicar-se em folhas de cultivares resistentes (Cowger \& Mundt, 2002).

O objetivo do presente trabalho foi avaliar o crescimento e a produção de primeiro e segundo ciclos de bananeiras das cultivares Prata-Anã em seis sistemas de plantio, e das cultivares Caipira e Thap Maeo sob três sistemas de plantio, nas condições edafoclimáticas do norte de Minas Gerais.

\section{MATERIAL E MÉTODOS}

O trabalho foi implantado em 2004, em solo classificado como Aluvial, heterogêneo e estratificado, apresentando redução do carbono orgânico em profundidade, conduzido na Fazenda Experimental do projeto "Crer-Ser", em parceria com a Universidade Estadual de Montes Claros (UNIMONTES), no município de Janaúba-MG. O município apresenta latitude de $15^{\circ}$ $48^{\prime} 09^{\prime \prime}$ sul, longitude $43^{\circ} 18^{\prime \prime} 32^{\prime}$, altitude de $533 \mathrm{~m}$ e médias anuais de precipitação de $900 \mathrm{~mm}$, de temperatura média de $25^{\circ} \mathrm{C}$ e de umidade relativa do ar de $56 \%$. O experimento foi instalado com mudas micropropagadas, cedidas pela Empresa Campo Biotecnologia. O sistema de irrigação utilizado foi por microaspersão, com turno de rega pré-fixado, baseado nos caracteres do solo, condição atmosférica e nas necessidades da bananeira. Os tratos culturais utilizados foram baseados em recomendações técnicas (Alves, 1999). O plantio das mudas em campo foi realizado no dia 03 de maio de 2004, no espaçamento de 3,0 m x 2,0 m, conduzindo-se cada família com mãe, filha e neta.

O delineamento experimental foi o de blocos casualizados, com quatro repetições e seis plantas úteis por parcela. Os tratamentos foram compostos pelas cultivares Prata-Anã, Caipira e Thap Maeo, em seis diferentes sistemas de plantio: 1)'PrataAnã' em sistema de plantio convencional (apenas 'Prata-Anã'); 2)'Prata-Anã' intercalada com 'Caipira' e 'Thap Maeo' (Figura 1); 3) 'Prata-Anã' com uma linha de bordadura de 'Caipira' (Figura 2); 4)'Prata-Anã' com duas linhas de bordaduras de 'Caipira'; 5)'Prata-Anã' com uma linha de bordadura de 'Thap Maeo' (Figura 2), e 6)'Prata-Anã' com duas linhas de bordaduras de 'Thap Maeo'.

Foram avaliadas as seguintes características de crescimento e produção: altura da planta obtida a partir do solo até a roseta foliar $(\mathrm{cm})$, circunferência do pseudocaule obtida na base da planta junto ao solo $(\mathrm{cm})$, número total de folhas emitidas do plantio até o florescimento, número de folhas vivas na colheita, número de dias do plantio à colheita, massa do cacho $(\mathrm{kg})$, produtividade $\left(\right.$ t.ha $\left.^{-1}\right)$, número de pencas e número de frutos por cacho.

Os dados foram analisados em duas etapas. Na primeira, verificou-se o efeito dos 6 tratamentos descritos anteriormente sobre a cultivar 'Prata-Anã'. Na segunda etapa, analisou-se considerando um esquema fatorial $2 \times 2+1$, sendo duas cultivares (Caipira e Thap Maeo), dois sistemas de bordadura (com uma e duas linhas) e um sistema de interplantio (Figura 1).

Os dados obtidos foram submetidos à análise de variância com desdobramentos das interações significativas, sendo os efeitos dos tratamentos comparados pelo teste de Tukey, a $5 \%$ de probabilidade. As análises estatísticas foram efetuadas com o auxílio dos programas SAS e SISVAR.

\section{RESULTADOS E DISCUSSÃO}

De acordo com a análise de variância, não houve interações significativas entre as cultivares e os sistemas de 
plantio, a 5\% de probabilidade, para a maioria das características avaliadas.

Na Tabela 1, são apresentados os resultados relativos às características de altura de plantas, circunferência do pseudocaule e número total de folhas no florescimento.

A altura das plantas estudadas variou de $180 \mathrm{~cm}$ na Caipira a $246 \mathrm{~cm}$ na Thap Maeo, no primeiro ciclo, e de $226 \mathrm{~cm}$ na 'Caipira' a $284 \mathrm{~cm}$ na 'Thap Maeo', no segundo ciclo (Tabela 1). A altura de plantas é considerada um dos principais descritores sob o ponto de vista fitotécnico e de melhoramento, pois influi nos aspectos de densidade de plantio, produção e manejo da cultura (Belalcázar Carvajal, 1991).

A cultivar Thap Maeo apresentou a maior média para a característica de circunferência do pseudocaule, no primeiro ciclo, com 66,4 cm (Tabela 1). A cultivar Caipira apresentou as menores médias para as características de altura de plantas e circunferência do pseudocaule, no primeiro e segundo ciclos.

A altura de plantas, o diâmetro de pseudocaule, associados à massa do cacho são características estreitamente relacionadas ao tombamento de planta pela ação dos ventos (Teixeira, 2001).

Não foram observadas diferenças significativas para a característica de número de folhas totais no florescimento entre as cultivares avaliadas e nos diferentes sistemas de plantio. Este também é considerado um descritor muito importante, pois as folhas constituem a parte essencial na geração de fotoassimilados por meio da taxa de fotossíntese da planta e que influencia diretamente nas características de crescimento e produção das variedades (Alves, 1999).

Dentre os caracteres estudados, os que expressam o vigor da planta, tais como, a altura e a circunferência do pseudocaule, apresentaram uma tendência de incremento do primeiro para o segundo ciclos, exceto a circunferência do pseudocaule, na cultivar Thap Maeo, independentemente do sistema de plantio. $\mathrm{O}$ maior incremento para altura de planta foi verificado na cultivar Caipira, com $23,7 \%$, e o menor para a cultivar Thap Maeo, com 18,1\%. Rodrigues et al. (2006), avaliando a cultivar Caipira na região norte de Minas Gerais, também observaram tendência de aumento da altura de plantas e do diâmetro do pseudocaule do primeiro para o segundo ciclos. Resultados similares também foram divulgados por Donato et al. (2006), indicando o aumento da altura de planta, circunferência de pseudocaule e número de folhas emitidas do primeiro para o segundo ciclo das cultivares Caipira e Thap Maeo no sudoeste da Bahia.

Na Tabela 2, são apresentados os resultados de produtividade, número de folhas vivas na colheita e ciclo da cultura. Independentemente do sistema de plantio, a cultivar Thap Maeo apresentou as maiores médias quando comparada à cultivar Caipira, para as características de produtividade e número de folhas vivas na colheita, no primeiro e segundo ciclos. A cultivar Thap Maeo apresentou produtividade média de 19,8 t/ha e 20,8 t/ha, no primeiro e segundo ciclos, respectivamente (Tabela 2). Em relação aos dois ciclos de produção, foi observado proporcionalmente maior incremento na produtividade para a cultivar Caipira, com 19,9\% e o menor na 'Thap Maeo', com
$5,0 \%$. Os resultados obtidos no presente trabalho indicam produtividades inferiores àquelas obtidas por Donato et al. (2006). A baixa produção observada nas cultivares avaliadas deve ter ocorrido em virtude das qualidades física e química do solo. Segundo Borges \& Oliveira (2000), a bananeira é uma planta muito exigente em nutrientes, principalmente potássio e nitrogênio, que, nas condições do presente trabalho, apresentaram níveis reduzidos de potássio e matéria orgânica no solo.

O número de folhas vivas na colheita variou de 7,7 na 'Caipira' a 10,1 na 'Thap Maeo', para o primeiro ciclo, e de 5,5 na 'Caipira' a 8,4 na 'Thap Maeo', para o segundo ciclo (Tabela 2). Quanto maior o número de folhas vivas ou funcionais durante o ciclo produtivo, maior potencial de produção terá a planta, já que essas folhas são responsáveis pela realização da fotossíntese e da translocação dos fotoassimilados destinados aos frutos (Pereira, 1997). Estudos realizados por Pereira et al. (2000) com a cultivar Prata-Anã em Jaíba, norte de Minas Gerais, apresentaram 8,7 folhas vivas na época da colheita, enquanto em Visconde do Rio Branco, região da Zona da Mata Mineira, apresentaram 7 folhas vivas (Pereira et al., 1999).

O ciclo da cultura foi de 426 dias na 'Thap Maeo' a 433 dias na 'Caipira', para o primeiro ciclo; e de 567 dias na 'Caipira' a 667 dias na 'Thap Maeo', para o segundo ciclo. O ciclo produtivo tende a se alterar em função de cada cultivar, da condição edafoclimática de cada região, do tipo de solo do local, dentre outros fatores. Segundo Pereira (1997), o conhecimento do ciclo da cultura, que varia principalmente de acordo com as características climáticas de cada região, a irrigação e a cultivar são importantes, pois, a partir desses dados, o agricultor poderá concentrar a produção em época de maior preço.

A massa dos cachos, o número de pencas e de frutos por cacho apresentaram diferenças significativas entre as cultivares estudadas (Tabela 3). Tais características apresentaram estreita relação, sendo que a massa do cacho variou de $4,40 \mathrm{~kg}$ e $4,25 \mathrm{~kg}$ na Caipira a $12,05 \mathrm{~kg}$ e 12,46 kg na Thap Maeo, para o primeiro e segundo ciclo respectivamente. A característica de número de pencas variou de 6,4 e 5,6 na 'Caipira' a 10,8 e 12,2 na 'Thap Maeo' para o primeiro e segundo ciclos respectivamente.

Existe uma estreita relação entre número de pencas e de frutos por cacho. As cultivares que apresentam elevado número de pencas, na maioria das vezes, apresentam também um grande número de frutos (Lima Neto et al., 2003). De acordo com Silva et al. (2002), o primeiro ciclo, não é adequado para analisar a massa do cacho para a maioria das cultivares de banana, uma vez que tal característica pode aumentar do primeiro para o segundo ciclo.

O número de frutos por cacho variou de 108 na cultivar Caipira a 189 na Thap Maeo, no primeiro ciclo, e de 88 na 'Caipira' para 201 frutos na 'Thap Maeo', para o segundo ciclo. Incrementos no número de frutos também foram obtidos por Donato et al. (2006), avaliando o comportamento de cultivares e híbridos de bananeira em dois ciclos de produção, no sudeste da Bahia. Estudos realizados com a cultivar Caipira no Norte de Minas Gerais apresentam incrementos no número de frutos a partir do segundo ciclo até o quarto ciclo avaliado, com média de 174 frutos por cacho, no segundo ciclo (Rogrigues et al., 2006). 
Os diferentes sistemas de plantio influenciaram significativamente nas características de número de folhas vivas na colheita no primeiro ciclo, sendo superior o plantio com duas fileiras de bordaduras. Produtividade, massa do cacho, número de pencas/cacho e número de folhas vivas na colheita, no segundo ciclo, o sistema com uma fileira de bordadura foi superior (Tabelas 1,2 e 3).

A análise da cultivar Prata-Anã, dentro dos seis sistemas de plantio, apresentou diferença significativa para as características número de folhas vivas na colheita, número de pencas/cacho e número de frutos/cacho, no segundo ciclo (Tabela 4). Embora não tenham sido detectadas diferenças significativas para outros caracteres avaliados, houve diferenças consideráveis principalmente para produtividade, se comparar o plantio com uma linha de bordadura ao sistema de plantio convencional. No primeiro e segundo ciclos, a diferença foi de $46 \%$ e $81 \%$ não-detectada pela estatística, mas foi observada uma média inferior para o sistema de plantio convencional da 'Prata-Anã' para a maioria das outras características avaliadas.
Resultados semelhantes foram obtidos por Nogueira (2005), em seis anos de avaliação, para três linhagens de café isoladas e em diferentes combinações de interplantio, em São Sebastião do Paraíso-MG, onde não foram observadas diferenças significativas quanto às características de vigor vegetativo, sendo que a variação ocorrida foi atribuída à interação da linhagem com o ambiente em que estava sendo cultivada. Já, para a característica de produtividade, foram observadas diferenças significativas, para diferentes proporções de misturas. Newton \& Swanston (1999), avaliando as misturas de variedades de cereais, como cevada, relatam muitos benefícios potenciais ao produtor, como: aumentos de rendimento entre 5 e $15 \%$, além de promover estabilidade da produção e na qualidade.

De acordo com Becker \& Leon (1998), o emprego de multilinhas, além de proporcionar maior durabilidade da resistência a alguns patógenos, fornece maior estabilidade na presença de variação para outros fatores ambientais.

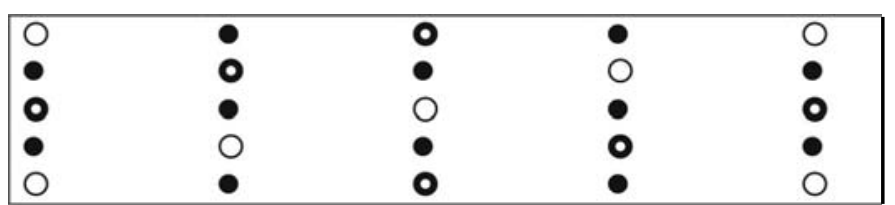

FIGURA 1 - Esquema do tratamento 2, com plantio intercalado de bananeiras, em Janaúba-MG

'Thap Maeo'

- 'Caipira'

- 'Prata-Anã'

\begin{tabular}{|llll|}
\hline 0 & 0 & 0 & 0 \\
0 & $\bullet$ & 0 & 0 \\
0 & $\bullet$ & $\bullet$ & 0 \\
0 & $\bullet$ & $\bullet$ & 0 \\
0 & 0 & 0 & 0 \\
\hline
\end{tabular}

FIGURA 2- Esquema dos tratamentos com uma linha de bordadura, em Janaúba-MG.

'Thap Maeo' ou 'Caipira'

- 'Prata-Anã'

TABELA 1 - Altura das plantas no florescimento $(\mathrm{cm})$, circunferência do pseudocaule $(\mathrm{cm})$ e número total de folhas no florescimento, das bananeiras 'Caipira' (CA) e 'Thap Maeo' (TM), em dois ciclos de cultivo, sob três sistemas de plantio, em JanaúbaMG, 2006.

\begin{tabular}{|c|c|c|c|c|c|c|c|c|c|}
\hline \multicolumn{10}{|c|}{ Primeiro ciclo } \\
\hline \multirow{3}{*}{$\begin{array}{c}\text { Sistemas } \\
\text { de } \\
\text { Plantio }^{2}\end{array}$} & \multicolumn{8}{|c|}{ Cultivares de banana } & \multirow[b]{2}{*}{ Médias } \\
\hline & $\mathrm{CA}$ & TM & Médias & $\mathrm{CA}$ & TM & Médias & $\mathrm{CA}$ & TM & \\
\hline & \multicolumn{3}{|c|}{$\begin{array}{c}\text { Altura da planta no florescimento } \\
(\mathrm{cm})\end{array}$} & \multicolumn{3}{|c|}{$\begin{array}{l}\text { Circunferência do pseudocaule no } \\
\text { florescimento }(\mathrm{cm})\end{array}$} & \multicolumn{3}{|c|}{$\mathrm{N}^{\circ}$ total de folhas no florescimento } \\
\hline 1 & $181 \mathrm{aB}$ & $250 \mathrm{aA}$ & 216 a & $50,3 \mathrm{aB}$ & $71,9 \mathrm{aA}$ & 61,1 a & $38,4 \mathrm{aA}$ & $37,0 \mathrm{aA}$ & 37,5 a \\
\hline 2 & $178 \mathrm{aB}$ & $243 \mathrm{aA}$ & $210 \mathrm{a}$ & $47,1 \mathrm{aB}$ & $60,8 \mathrm{aA}$ & 54,0 a & $39,2 \mathrm{aA}$ & $38,8 \mathrm{aA}$ & 39,0 a \\
\hline 3 & & & $210 \mathrm{a}$ & & & 55,2 a & & & 37,6 a \\
\hline Médias & $180 \mathrm{~B}$ & $246 \mathrm{~A}$ & & 48,7 B & $66,4 \mathrm{~A}$ & & 38,8 A & $37,7 \mathrm{~A}$ & \\
\hline CV (\%) & & & 14,00 & & & 14,80 & & & 4,68 \\
\hline \multicolumn{10}{|c|}{ Segundo ciclo } \\
\hline \multirow{3}{*}{$\begin{array}{l}\text { Sistemas } \\
\text { de } \\
\text { Plantio }^{2}\end{array}$} & \multicolumn{8}{|c|}{ Cultivares de banana } & \\
\hline & CA & TM & Médias & $\mathrm{CA}$ & TM & Médias & CA & TM & Médias \\
\hline & \multicolumn{3}{|c|}{$\begin{array}{l}\text { Altura da planta no florescimento } \\
(\mathrm{cm})\end{array}$} & \multicolumn{3}{|c|}{$\begin{array}{l}\text { Circunferência do pseudocaule no } \\
\text { florescimento }(\mathrm{cm})\end{array}$} & \multicolumn{3}{|c|}{$\mathrm{N}^{\circ}$ de folhas total no florescimento } \\
\hline 1 & $229 \mathrm{aA}$ & $286 a A$ & 257 a & $52,4 \mathrm{aA}$ & $65,9 \mathrm{aA}$ & 59,2 a & $34,5 \mathrm{aA}$ & $31,5 \mathrm{aA}$ & 33,0 a \\
\hline 2 & $223 \mathrm{aA}$ & $282 \mathrm{aA}$ & $253 a$ & $50,2 \mathrm{aA}$ & $63,6 \mathrm{aA}$ & 56,9 a & $37,4 \mathrm{aA}$ & $38,0 \mathrm{aA}$ & 37,7 a \\
\hline 3 & & & 257 a & & & 57,8 a & & & 38,2 a \\
\hline Médias & $226 \mathrm{~A}$ & $284 \mathrm{~A}$ & & $51,3 \mathrm{~A}$ & $64,7 \mathrm{~A}$ & & 34,7 A & 35,9 A & \\
\hline CV (\%) & & & 24,01 & & & 20,54 & & & 14,62 \\
\hline
\end{tabular}

${ }^{1}$ Médias seguidas da mesma letra maiúscula nas linhas e minúscula nas colunas, dentro da mesma característica, não diferem entre si, a $5 \%$ de probabilidade, pelo teste de Tukey.

${ }^{2}$ Sistemas de plantio: 1- Uma fileira de bordadura; 2- Duas fileiras de bordaduras, e 3- interplantio. 
TABELA 2 - Produtividade, número de folhas vivas na colheita e número de dias do plantio à colheita das bananeiras 'Caipira' (CA) e 'Thap Maeo' (TM), em dois ciclos de cultivo, sob três sistemas de plantio, em Janaúba-MG, 2006 '.

\begin{tabular}{|c|c|c|c|c|c|c|c|c|c|}
\hline \multirow{2}{*}{\multicolumn{10}{|c|}{ Primeiro ciclo }} \\
\hline \multirow{3}{*}{$\begin{array}{c}\text { Sistemas } \\
\text { de } \\
\text { Plantio }\end{array}$} & \multicolumn{6}{|c|}{ Cultivares de banana } & & & \\
\hline & $\mathrm{CA}$ & TM & Médias & $\mathrm{CA}$ & TM & Médias & $\mathrm{CA}$ & TM & Médias \\
\hline & \multicolumn{3}{|c|}{ Produtividade $(\mathrm{t} / \mathrm{ha})$} & \multicolumn{3}{|c|}{$\mathrm{N}$ de folhas vivas na colheita } & \multicolumn{3}{|c|}{$\mathrm{N}$ de dias do plantio-colheita } \\
\hline 1 & $6,3 \mathrm{aB}$ & $19,5 \mathrm{aA}$ & 12,9 a & $6,6 \mathrm{bB}$ & $9,2 \mathrm{bA}$ & $7,9 \mathrm{~b}$ & $431 \mathrm{aA}$ & $430 \mathrm{aA}$ & 431 a \\
\hline 2 & $8,3 \mathrm{aB}$ & $20,1 \mathrm{aA}$ & 14,2 a & $8,8 \mathrm{aB}$ & $11,1 \mathrm{aA}$ & 10,0 a & $434 \mathrm{aA}$ & $422 \mathrm{aA}$ & $428 \mathrm{a}$ \\
\hline 3 & & & 11,5 a & & & $8,9 \mathbf{a b}$ & & & $440 \mathrm{a}$ \\
\hline Médias & $7,3 \mathrm{~B}$ & $19,8 \mathrm{~A}$ & & $7,7 \mathrm{~B}$ & 10,1 A & & $433 A$ & $426 \mathrm{~A}$ & \\
\hline CV (\%) & & & 48,46 & & & 11,11 & & & 5,95 \\
\hline \multicolumn{10}{|c|}{ Segundo ciclo } \\
\hline \multirow{3}{*}{$\begin{array}{c}\text { Sistemas } \\
\text { de } \\
\text { Plantio }^{2} \\
\end{array}$} & \multicolumn{9}{|c|}{ Cultivares de banana } \\
\hline & $\mathrm{CA}$ & TM & Médias & $\mathrm{CA}$ & TM & Médias & $\mathrm{CA}$ & TM & Médias \\
\hline & \multicolumn{3}{|c|}{ Produtividade $(\mathrm{t} / \mathrm{ha})$} & \multicolumn{3}{|c|}{$\mathrm{N}$ de folhas vivas na colheita } & \multicolumn{3}{|c|}{$\mathrm{N}$ de dias do plantio-colheita } \\
\hline 1 & $9,9 \mathrm{aB}$ & $26,3 \mathrm{aA}$ & 18,1 a & $5,0 \mathrm{aB}$ & $8,8 \mathrm{aA}$ & 6,9 a & $583 a A$ & $683 a A$ & 601 a \\
\hline $\begin{array}{l}2 \\
3\end{array}$ & $7,6 \mathrm{aB}$ & $15,2 \mathrm{bA}$ & $\begin{array}{c}11,4 \mathrm{~b} \\
15,2 \mathrm{ab}\end{array}$ & $6,0 \mathrm{aA}$ & $8,0 \mathrm{aA}$ & $\begin{array}{l}7,0 \text { a } \\
5,2 \quad \mathbf{a}\end{array}$ & $551 \mathrm{aA}$ & $650 a A$ & $\begin{array}{l}633 a \\
666 a\end{array}$ \\
\hline Médias & 8,75 B & $20,8 \mathrm{~A}$ & & $5,5 \mathrm{~B}$ & $8,4 \mathrm{~A}$ & & $567 \mathrm{~A}$ & $667 \mathrm{~A}$ & \\
\hline CV (\%) & & & 23,77 & & & 24,36 & & & 15,34 \\
\hline
\end{tabular}

${ }^{1}$ Médias seguidas da mesma letra maiúscula nas linhas e minúscula nas colunas, dentro da mesma característica, não diferem entre si, a $5 \%$ de probabilidade, pelo teste de Tukey. ${ }^{2}$ Sistemas de plantio: 1- Uma fileira de bordadura; 2- Duas fileiras de bordaduras, e 3-interplantio.

TABELA 3 - Peso do cacho, número de pencas por cacho e número de frutos por cacho das bananeiras 'Caipira' (CA) e 'Thap Maeo' (TM), em dois ciclos de cultivo, sob três sistemas de plantio, em Janaúba-MG, $2006^{1}$.

\begin{tabular}{|c|c|c|c|c|c|c|c|c|c|}
\hline \multicolumn{10}{|c|}{ Primeiro ciclo } \\
\hline \multirow{3}{*}{$\begin{array}{c}\text { Sistemas } \\
\text { de } \\
\text { Plantio }^{2}\end{array}$} & \multicolumn{9}{|c|}{ Va riedades de banana } \\
\hline & $\mathrm{CA}$ & TM & Médias & CA & TM & Médias & $\mathrm{CA}$ & TM & Médias \\
\hline & \multicolumn{3}{|c|}{ Peso do cacho $(\mathrm{kg})$} & \multicolumn{3}{|c|}{ de pencas por cacho } & \multicolumn{3}{|c|}{ de frutos por cacho } \\
\hline 1 & $3,81 \mathrm{aB}$ & $11,68 \mathrm{aA}$ & $7,74 \mathrm{a}$ & $5,9 \mathrm{aB}$ & $10,7 \mathrm{aA}$ & 8,3 a & $100 a B$ & $178 \mathrm{aA}$ & $139 \mathbf{a}$ \\
\hline $\begin{array}{l}2 \\
3\end{array}$ & $5,00 \mathrm{aB}$ & $12,42 \mathrm{aA}$ & $\begin{array}{l}8,71 \mathrm{a} \\
6,54 \mathrm{a}\end{array}$ & $7,0 \mathrm{aB}$ & $11, \mathrm{OaA}$ & $\begin{array}{l}9,0 \text { a } \\
7,7 \text { a }\end{array}$ & $116 \mathrm{aB}$ & $201 \mathrm{aA}$ & $\begin{array}{l}158 \mathrm{a} \\
122 \mathrm{a}\end{array}$ \\
\hline Médias & $4,40 \mathrm{~B}$ & $12,05 \mathrm{~A}$ & & $6,4 \mathrm{~B}$ & $10,8 \mathrm{~A}$ & & $108 \mathrm{~B}$ & $189 \mathrm{~A}$ & \\
\hline CV (\%) & & & 48,26 & & & 12,35 & & & 28,74 \\
\hline \multicolumn{10}{|c|}{ Segundo ciclo } \\
\hline \multirow{3}{*}{$\begin{array}{c}\text { Sistemas } \\
\text { de } \\
\text { Plantio }^{2}\end{array}$} & \multicolumn{9}{|c|}{ Variedades de banana } \\
\hline & CA & TM & Médias & CA & TM & Médias & CA & TM & Médias \\
\hline & \multicolumn{3}{|c|}{ Peso do cacho $(\mathrm{kg})$} & \multicolumn{3}{|c|}{$\mathrm{N}$ de pencas por cacho } & \multicolumn{3}{|c|}{$\mathrm{N}$ de frutos por cacho } \\
\hline 1 & $5,95 \mathrm{aB}$ & $15,78 \mathrm{aA}$ & $\mathbf{1 0 , 8 6}$ a & $6,0 \mathrm{aB}$ & $13,9 \mathrm{aA}$ & $\mathbf{1 0 , 0}$ a & $93 \mathrm{aB}$ & $237 \mathrm{aA}$ & 165 a \\
\hline $\begin{array}{l}2 \\
3\end{array}$ & $4,56 \mathrm{aB}$ & $9,15 \mathrm{bA}$ & $\begin{array}{l}6,85 \text { b } \\
9,1 \mathrm{ab}\end{array}$ & $5,2 \mathrm{aB}$ & $10,5 \mathrm{bA}$ & $\begin{array}{l}7,9 \text { b } \\
9,8 \mathbf{a b}\end{array}$ & $79 \mathrm{aB}$ & $165 \mathrm{bA}$ & $\begin{array}{l}122 \mathrm{~b} \\
153 \mathrm{ab}\end{array}$ \\
\hline Médias & $4,25 \mathrm{~B}$ & $12,46 A$ & & $5,6 \mathrm{~B}$ & $12,2 \mathrm{~A}$ & & $88 \mathrm{~B}$ & $201 \mathrm{~A}$ & \\
\hline CV (\%) & & & 23,78 & & & 11,99 & & & 20,20 \\
\hline
\end{tabular}

${ }^{1}$ Médias seguidas da mesma letra maiúscula nas linhas e minúscula nas colunas, dentro da mesma característica, não diferem entre si, a $5 \%$ de probabilidade, pelo teste de Tukey. ${ }^{2}$ Sistemas de plantio: 1- Uma fileira de bordadura; 2- Duas fileiras de bordaduras, e 3 - interplantio.

TABELA 4 - Altura da planta no florescimento (ALTF), circunferência da base do pseudocaule no florescimento (CPB), número de folhas totais até o florescimento (NFTF), produtividade (PTOD), número de folhas vivas na colheita (NFVC), número de dias do plantio à colheita (NDPC), massa do cacho (MC), número de pencas/cacho (NP), e número de frutos/cacho (NFRC) da bananeira 'Prata-Anã' em diferentes sistemas de plantio, em Janaúba-MG, 2006.

\begin{tabular}{|c|c|c|c|c|c|c|c|c|c|}
\hline \multicolumn{10}{|c|}{ Primeiro ciclo } \\
\hline Trat & $\begin{array}{c}\text { ALTF } \\
(\mathrm{cm})\end{array}$ & $\begin{array}{l}\text { CPB } \\
(\mathrm{cm})\end{array}$ & $\begin{array}{c}\text { NFTF } \\
\text { (unidade) }\end{array}$ & $\begin{array}{c}\text { PROD } \\
(\mathrm{t} / \mathrm{ha})\end{array}$ & $\begin{array}{c}\text { NFVC } \\
\text { (unidade) }\end{array}$ & $\begin{array}{c}\text { NDPC } \\
\text { (unidade) }\end{array}$ & $\begin{array}{l}\mathrm{MC} \\
(\mathrm{kg})\end{array}$ & $\begin{array}{c}\text { NP } \\
\text { (unidade) }\end{array}$ & $\begin{array}{c}\text { NFRC } \\
\text { (unidade) }\end{array}$ \\
\hline 1 & $191.71 \mathrm{a}$ & $65,33 a$ & $41.7 \mathrm{a}$ & $10,75 a$ & $7.7 \mathrm{a}$ & $411.7 \mathrm{a}$ & $6,46 a$ & $8,0 \mathrm{a}$ & $111,1 \mathrm{a}$ \\
\hline 2 & $191.37 \mathrm{a}$ & $66,31 \mathrm{a}$ & $41.4 \mathrm{a}$ & $11,53 \mathrm{a}$ & $9.6 \mathrm{a}$ & $437.4 \mathrm{a}$ & $6,92 a$ & $7,7 \mathrm{a}$ & $114,4 a$ \\
\hline 3 & $200.87 a$ & $70,54 a$ & $39.6 \mathrm{a}$ & $15,71 \mathrm{a}$ & $11.5 \mathrm{a}$ & $409.9 a$ & $9,43 a$ & $8,8 \mathrm{a}$ & $131,7 a$ \\
\hline 4 & $203.17 \mathrm{a}$ & $70,12 \mathrm{a}$ & $39.7 \mathrm{a}$ & $15,22 \mathrm{a}$ & $10.7 \mathrm{a}$ & $416.4 \mathrm{a}$ & $9,14 \mathrm{a}$ & $8,9 \mathrm{a}$ & $125,3 \mathrm{a}$ \\
\hline 5 & $198.79 \mathrm{a}$ & $75,29 \mathrm{a}$ & $43.8 \mathrm{a}$ & $15,26 a$ & $9.6 \mathrm{a}$ & $440.0 \mathrm{a}$ & $9,16 a$ & $8,7 a$ & $135,6 \mathrm{a}$ \\
\hline 6 & $205.50 a$ & $69,37 a$ & $41.6 a$ & $13,28 \mathrm{a}$ & $10.6 a$ & $429.1 \mathrm{a}$ & $7,97 a$ & $8,8 \mathrm{a}$ & $131,6 a$ \\
\hline Médias & 198,57 & 58,42 & 41,3 & 13,63 & 9,9 & 424,1 & 8,18 & 8,48 & 124,9 \\
\hline CV (\%) & 11,44 & 10,23 & 5,73 & 30,91 & 18,56 & 3,93 & 30,91 & 9,29 & 14,50 \\
\hline \multicolumn{10}{|c|}{$\begin{array}{l}\text { Segundo ciclo } \\
\end{array}$} \\
\hline Trat & $\begin{array}{c}\text { ALTF } \\
(\mathrm{cm})\end{array}$ & $\begin{array}{l}\text { CPB } \\
(\mathrm{cm})\end{array}$ & $\begin{array}{c}\text { NFTF } \\
\text { (unidade) }\end{array}$ & $\begin{array}{c}\text { PROD } \\
(\mathrm{t} / \mathrm{ha})\end{array}$ & $\begin{array}{c}\text { NFVC } \\
\text { (unidade) }\end{array}$ & $\begin{array}{c}\text { NDPC } \\
\text { (unidade) }\end{array}$ & $\begin{array}{l}\text { MC } \\
(\mathrm{kg})\end{array}$ & $\begin{array}{c}\text { NP } \\
\text { (unidade) }\end{array}$ & $\begin{array}{c}\text { NFRC } \\
\text { (unidade) }\end{array}$ \\
\hline 1 & $242,12 a$ & $79,17 \mathrm{a}$ & $48,1 \mathrm{a}$ & $14,22 \mathrm{a}$ & $8,2 \mathrm{~b}$ & $534,8 \mathrm{a}$ & $8,54 a$ & $7,9 b$ & $115,8 \mathrm{~b}$ \\
\hline 2 & $240,41 \mathrm{a}$ & $76,97 a$ & $43,6 a$ & $17,92 \mathrm{a}$ & $10,0 a b$ & $619,9 a$ & $10,76 a$ & $8,9 \mathrm{ab}$ & $133,4 \mathrm{ab}$ \\
\hline 3 & $253,62 a$ & $82,92 a$ & $44,3 a$ & $25,71 \mathrm{a}$ & $10,4 \mathrm{ab}$ & $610,8 \mathrm{a}$ & $15,43 a$ & $10,4 a$ & $87,7 \mathrm{a}$ \\
\hline 4 & $235,75 a$ & $76,00 \mathrm{a}$ & $42,5 a$ & $16,86 a$ & $9,6 \mathrm{ab}$ & $677,4 a$ & $10,12 \mathrm{a}$ & $8,6 \mathrm{ab}$ & $124,7 \mathrm{ab}$ \\
\hline 5 & $238,83 a$ & $77,00 \mathrm{a}$ & $46,4 a$ & $17,84 a$ & $10,2 \mathrm{ab}$ & $591,7 a$ & $10,71 \mathrm{a}$ & $8,3 \mathrm{ab}$ & $132,6 a b$ \\
\hline 6 & $255,00 \mathrm{a}$ & $81,87 a$ & $43,4 a$ & $19,54 \mathrm{a}$ & $11,0 \mathrm{a}$ & $641,7 a$ & $11,73 \mathrm{a}$ & $8,9 \mathrm{ab}$ & $133,4 \mathrm{ab}$ \\
\hline Médias & 244,29 & 78,99 & 44,7 & 18,68 & 9,9 & 612,7 & 11,21 & $\mathbf{8 , 8 3}$ & 137,9 \\
\hline CV $(\%)$ & 14,73 & 14,09 & 7,53 & 35,46 & 9,60 & 10,54 & 35,48 & 13,80 & 22,61 \\
\hline
\end{tabular}

${ }^{1}$ Médias seguidas da mesma letra na coluna não se diferem, a $5 \%$ de probabilidade, pelo teste de Tukey. ${ }^{2}$ Tratamentos: 1 : Testemunha, plantio exclusivo da 'Prata-Anã'; 2: 'Prata-Anã' intercalado com variedade Thap Maeo e Caipira; 3: 'Prata-Anã' com uma bordadura de 'Thap Maeo; 4: 'Prata-Anã' com duas linhas de bordadura 'Thap Maeo'; 5: 'Prata-Anã com' 1 linha de bordadura de 'Caipira'; 6: 'Prata-Anã' com duas linhas de bordadura de 'Caipira'. 


\section{CONCLUSÕES}

1- Os sistemas de plantio utilizados não influenciam nas características de crescimento, no primeiro e segundo ciclos para as três cultivares avaliadas;

2- A cultivar Thap Maeo é superior à Caipira no primeiro e segundo ciclos, para a maioria das características de crescimento e produção no norte de Minas Gerais.

\section{REFERÊNCIAS}

AGRIANUAL. Banana. São Paulo: FNP, Consultoria e Agroinformativos, 2005. p. 220-229.

ALVES, E. J. A cultura da banana: aspectos técnicos, socioeconômicos e agroindustriais. 2 ed. Brasília: Embrapa-SPI/ Cruz das Almas: Embrapa-CNPMF, 1999. 585 p.

BECKER, H.C.; LEON, J. Stability analysis in plant breeding. Plant Breeding, Berlin, v.101, n.1, p.1-23, 1998.

BELALCÁZAR CARVAJAL, S.L. El cultivo del plátano em el trópico. Cali: Impressora Feriva, 1991.376p.

BORGES, A. L.; OLIVEIRA, AM. G. Nutrição calagem e adubação. In: EMBRAPA. Banana produção: aspectos técnicos. Brasília: Embrapa Comunicação para Transferência de Tecnologia, 2000.143 p.

CASTRO, M.E.A,; PEREIRA, J.C.R.; GASPAROTTO, L.Primeiro relato de ocorrência da Sigatoka-negra em Minas Gerais. Fitopatologia Brasileira, Brasília, v.30, n.6,p.668, 2005.

COWGER, C.; MUNDT, C.C. Effects of Wheat Cultivar Mixtures on Epidemic Progression of Septoria tritici Blotch and Pathogenicity of Mycosphaerella graminicola. Phytopathology, St. Paul, v.92, n.6, p.621-625, 2002.

DONATO, S.L.R.; SILVA, S.O.; LUCCA FILHO, O.A.; LIMA, M.B.; DOMINGUES, H.; ALVES, J.S. Comportamento de variedades e híbridos de bananeira (Musa spp.), em dois ciclos de produção no sudeste da Bahia. Revista Brasileira de Fruticultura, Jaboticabal, v. 28, n. 1, 2006.

FAO. Food and Agricultural Organization. Disponível em: $<$ http:/ /apps.fao.org>. Acesso em: 18 jul 2007.

GONÇALVES, J.S.; PEREZ, L.H.; SOUZA, S.A.M. Perspectiva econômica da banana não é negra e futuro indica: siga a modernidade e toca inovação. In: REUNIÃO ITINERANTE DE FITOSSANIDADE DO INSTITUTO BIOLÓGICO, 13., 2005, Registro. p.1-15.

LIMANETO, F. P. ; SILVA, S. de O. e ; FLORES, J. C. O. ; JESUS, O. N. de; PAIVA, L. E. Relações entre caracteres de rendimento e de desenvolvimento em genótipos de bananeira. Magistra,
Cruz das Almas, v. 15, n. 2, p. 275-281, 2003.

NEWTON A.C.; SWANSTON J.S. Cereal variety mixtures reducing inputs and improving yield and quality: why isn't everybody growing them? Dundee, UK: Scottish Crop Research Institute, 1999. p.55-59. (Annual Report for 1998/99)

NOGUEIRA, A.M. Avaliação da produtividade e vigor vegetativo de linhagens das cultivares catuai-vermelho e catuai-amarelo (Coffea arábica L.) Plantadas individualmente e em diferentes combinações. Revista Ciência e Agrotenologia, Lavras, v. 29, n. 1, p.2277-3333, 2005.

PEREIRA, M.C.T.Crescimento e produção de primeiro ciclo da bananeira (Musa spp.)'Prata-Anã' (AAB) em sete espaçamentos, em Jaíba e Visconde do Rio Branco-MG. 1997.56f. Dissertação (Mestrado em Fitotecnia) - Universidade Federal de Viçosa, Viçosa, 1997.

PEREIRA, M.C.T.; SALOMAO, L.C.C.; SILVA, S.O.; SEDIYAMA, C.S.; COUTO, F.A.D'A.; SILVA NETO, S.P. Crescimento e produção de primeiro ciclo da bananeira (Musa spp.)'Prata-Anã' (AAB) em sete espaçamentos, em Jaíba, MG. Pesquisa Agropecuária Brasileira, Brasília, v. 35, p. 1377-1387, 2000.

PEREIRA, M.C.T.; SALOMAO, L.C.C.; SILVA, S.O.; SEDIYAMA, C.S.; SILVA NETO, S.P.; COUTO, F.A.D'A.; Crescimento e produção de primeiro ciclo da bananeira (Musa spp.)'Prata Anã' (AAB) em sete espaçamentos, em Visconde do Rio Branco-MG. Revista Ceres, Viçosa, v. 46, n.263, p. 53-66, 1999.

RODRIGUES, M.G.V.; SOUTO, R.F.; SILVA, S.O. Avaliação de genótipos de bananeira sob irrigação. Revista Brasileira de Fruticultura, Jaboticabal, v.28, n.3, p.444-448, 2006.

SECEX. Secretaria de Comércio Exterior Ministério do Desenvolvimento, Indústria e Comércio (MDIC), 2006: Disponível: $<$ http://www.mdic.gov.br>.

SILVA, S. O.; SANTOS-SEREJO, J. A. dos. Melhoramento da bananeira para resistência: resultados obtidos pelo melhoramento convencional. In: SIMPÓSIO BRASILEIRO SOBRE BANANICULTURA E WORKSHOP DO GENOMA MUSA, 5 ., 2003, Paracatu. Anais... Cruz das Almas: Nova Civilização, 2003. p. 28-34.

SILVA, S. O.; ALVES, E.J. LIMA, M.B.; SILVEIRA, J.R.S. Bananeira. In: BRUCKNER C.H.(Ed.). Melhoramento de fruteiras tropicais. Viçosa: UFV, 2002. p. 101-157.

SILVA, S. O.; ROCHA, S.A.; ALVES, E.J.; CREDICO, M. DI.; PASSOS, A.R. Caracterização morfológica e avaliação de cultivares e hibridos de bananeira. Revista Brasileira de Fruticultura, Jaboticabal, v. 22, n. 2, p. 161-169, agosto, 2000.

TEIXEIRA, L.A.J. Cultivares de bananeira. In: Ruggiero, C. (Coord.). Bananicultura. Jaboticabal: FUNEP, 2001. p. 150-170. 